\title{
La mise en marque des villes et des régions en France : la gouvernance comme facteur clé de succès
}

\author{
Charles-Édouard Houllier-Guibert ${ }^{\mathrm{a}}$, Anne Miriel $^{\mathrm{b}}$
}

RÉSUMÉ. Nombreux sont les territoires en France qui ont organisé ces dernières années la gestion de l’image officielle d'un territoire à l'échelle des métropoles ou des régions. Plusieurs ont échoué, mais d'autres sont parvenus à les lancer grâce à la gouvernance mise en place autour de l'objet «marque ». Il reste à pérenniser ces marques, outil de dynamisation collective des parties prenantes qui deviennent acteurs territoriaux. Cet article décrit la dimension relationnelle d'agents économiques, culturels ou sociaux, accompagnés par les services publics de l'administration locale et qui ont comme point commun la quête d'une dynamique territoriale.

\begin{abstract}
$\boldsymbol{A B S T R A C T}$. In recentyears, many territories in France bave organized the management of the official image of metropolises or regions. Several failed, but others managed to launch them thanks to the governance set up around the Brand. The perpetuation of these brands, as a tool for collective empowerment of stakeholders, is an important challenge based on good governance. This article describes the relational dimension of economic, cultural or social agents, accompanied by the public services of the local administration and which share the quest for a territorial mobilization.
\end{abstract}

Cet article propose d'étudier un nouveau dispositif qu'est la marque Territoire et qui vient relancer les pratiques de marketing des territoires au sein des collectivités locales françaises, principalement les plus grandes villes et les régions. Il convient en préambule de définir les deux termes qui forment un objet particulièrement lié à l'étude des jeux d'acteurs au sein du management public.

Le marketing des territoires a longtemps correspondu à l'ensemble des pratiques des acteurs publics (et parfois privés) organisées de manière stratégique ou opportune en matière de valorisation des espaces, d'attraction de divers flux (financiers, habitants, entreprises, touristes, événements), d'amélioration de l'image et plus largement en faveur du développement local. Sans qu'il y ait réellement de controverse sur les définitions, nous pouvons nous cantonner à celle qui tend à faire consensus chez les professionnels : «l'effort collectif de valorisation et d'adaptation des territoires à des marchés concurrentiels pour influencer, en leur faveur, le comportement des publics visés par une offre dont la valeur perçue est durablement supérieure à celles des concurrents » (Gollain, 2014). Le chercheur Camille Chamard propose une définition proche en tant que « démarche qui vise à élaborer, sur la base de la connaissance de l'environnement (géographique, démographique, culturel, économique, social, politique), l'offre territoriale", en précisant alors la finalité des marques Territoire «pour les collectivités territoriales (qui) est réellement d'engager une action stratégique conduisant à la définition d'un positionnement pour leur territoire afin de développer leur capital marque » (Chamard, 2014). Il s'agit bien, dans les deux cas, du volontarisme d'une multitude d'acteurs qui font territoire.

La marque Territoire dont il est question ici vient relancer et aussi transformer les pratiques, permettant notamment de couvrir les trois phases théoriques du marketing (Houllier-Guibert, 2012), là où auparavant les moyens budgétaires des territoires se cantonnaient principalement à la phase opérationnelle. La marque Territoire peut se définir comme la mise en œuvre par

\footnotetext{
a Docteur en géographie, maittre de conférences en « Stratégie et Territoire » à l’Université de Rouen, laboratoire NIMEC

${ }^{\mathrm{b}}$ Présidente, fondatrice d'Inkipit, agence spécialisée en marketing territorial
} 
l'acteur public local d'un choix stratégique (ciblage, positionnement) pensé collectivement et à moyen terme, à partir d'une collecte d'information plurielle, impliquant un ensemble d'acteurs locaux issus de plusieurs secteurs d'activité et qui ont comme point commun d'être attachés au territoire qui va être promu. Ce choix stratégique n'est pas figé et la marque, une fois lancée, est repensée de manière récurrente. Nous dissocions ici la marque Territoire de la marque territoriale en ce que la première est un bien commun, support de l'attractivité territoriale, tandis que la seconde, mise en place par des organisations privées, s'inscrit dans des logiques de consommation ou d'ancrage territorial (Houllier-Guibert, 2018). La marque Territoire vise le citoyen dudit territoire ou bien des agents extérieurs divers : économiques venant investir et s'implanter; touristiques venant dépenser leurs devises; habitants venant résider sur un temps long et ainsi animer la vie locale; étudiants, passant ponctuellement dynamiser le territoire ou bien restant pour y vivre.

Les collectivités locales sont aujourd'hui nombreuses à vouloir créer leur marque Territoire. Et les recompositions territoriales ${ }^{1}$, qui s'opèrent en conséquence de plusieurs lois, viennent renforcer le sentiment d'urgence exprimé par les élus de se doter d'une nouvelle identité fédératrice. S'agit-il d'une marque Territoire comme identité pour affirmer la légitimité d'un nouveau périmètre institutionnel? Ou sert-elle à fédérer élus, forces vives et habitants autour d'un nouveau projet de développement territorial avec la volonté de rendre ce territoire plus attractif? Sans être contradictoires, ces deux objectifs ne revêtent pas le même sens, et les démarches à mettre en œuvre pour les atteindre sont plutôt différentes.

La «marque institutionnelle » a pour objet de représenter la collectivité et les politiques publiques qu'elle conduit dans le cadre des compétences qui lui sont dévolues. Elle fédère les élus et agents de la collectivité qui travaillent ensemble à la mise en œuvre du projet politique. Sa réalisation relève d'un exercice classique de conception d'une identité visuelle et du mode d'emploi qui l'accompagne sous la forme d'une charte graphique.

La marque Territoire est une expression du projet de territoire et a pour objet de représenter tout ce qui le constitue ou contribue à sa réalisation; elle incarne une action collective au bénéfice du développement et de l'attractivité du territoire, portée par l'ensemble des acteurs publics, privés, habitants qui entendent y contribuer. Elle s'affranchit des périmètres institutionnels et des catégories d'acteurs, car elle a vocation à fédérer autour d'un objectif commun et partagé.

Cette vocation collaborative implique que sa réalisation relève d'un exercice plus complexe que la conception d'un objet graphique. Il s'agit d'engager une démarche structurante pour construire collectivement une stratégie de marketing territorial et la marque qui soutiendra sa mise en œuvre. À l'urgence du commanditaire s'oppose le temps long de l'amorçage d'une démarche engageante; à la volonté de se lancer dans un exercice rapidement visible de communication s'oppose le préalable de la mobilisation et du rassemblement des acteurs locaux autour d'une ambition partagée.

Dès lors, comment réunir les conditions qui permettent la réussite de la marque Territoire? La complexité de la démarche tient à la nécessité de mobiliser et d'impliquer un nombre très important d'acteurs, publics et privés qui, par la force du temps, des pratiques en cours et des habitudes, cultivent l'entre soi et le travail en silo. Au regard des pratiques observées par un chercheur dans de nombreuses créations de marques Territoire en France, associées au retour d'expérience d'une professionnelle qui accompagne aujourd'hui des collectivités après avoir fabriqué l'une des premières marques Région en France, nous postulons que la réussite passe par la mise en place d'un système organisationnel qui permette d'instaurer une relation constructive de concertation, d'échange et de travail avec les parties prenantes : c'est le management de la marque Territoire. Cet objet vise à recouvrir la vision et l'ambition du projet territorial, à travers les éléments constituant le socle fédérateur de l'identité du territoire, en tant que construction sociale. Ainsi, la marque Territoire incarne le positionnement marketing et l'offre qui en découle. Différents signes d'expression qui composent la marque se retrouvent dans un guide d'utilisation à l'usage des acteurs pour une pratique commune de la diffusion de l'image du territoire. Précisons que cet objectif est à peine atteint, la pérennité des marques étant difficile à obtenir en France, une fois leur lancement réalisé (HoullierGuibert et al., 2018).

La nécessité de construire avec méthode, et dans le temps nécessaire, le mode de management et d'organisation ad hoc pour faciliter la création d'une marque, fait basculer l'amorçage des démarches de marketing 
territorial vers une réorganisation de l'appareillage public. Cette transformation dépasse les enjeux d'image, dépasse aussi les enjeux politiques de mobilisation, pour mener vers ce que l'on nomme la conduite du changement et qui est en réalité, dans toute organisation, bien délicate à mener.

La constitution de la gouvernance nécessaire pour répondre à ce triple enjeu de l'image, de la mobilisation et de la conduite du changement, prend en compte plusieurs paramètres :

- le contexte institutionnel et les éventuelles recompositions spatiales en cours;

- le niveau de dialogue établi entre les acteurs publics et privés et notamment la relation avec les chefs d'entreprise;

- la capacité des acteurs locaux à travailler en réseau et en transversalité;

- la capacité à travailler en mode projet au sein de l'institution et avec les partenaires.

Ces paramètres interrogent les rapports de force, notamment politiques, ce que l'on appelle les jeux d'acteurs territoriaux, dont le bon équilibre est le facteur clé de réussite de la démarche de marketing qui s'entreprend. Une gouvernance s'organise alors autour de la marque Territoire. Si l'amélioration de l'image est une visée souhaitée par les responsables territoriaux, la poursuite d'un objectif intermédiaire, plus facile- ment mesurable, explique la large mobilisation des acteurs engagée à l'échelle d'une métropole, d'une région ou d'un département. Il s'agit de la construction collective de l'offre territoriale. Cette approche marketing par l'offre constitue une manière d'incarner la marque Territoire. Les acteurs mobilisés pensent le territoire où ils vivent ou travaillent, comme un objet qui peut être désirable, qui doit disposer d'un avantage comparatif, servir des occasions d'affaires ou tout type de proposition qui le rend attractif. Les démarches qui ne visent que l'image sont souvent amenées à décliner tandis que la construction de l'offre du territoire est une transformation et un ajustement permanent qui nécessite le maintien des échanges, de la construction mais aussi des tensions et des latences. L'objectif intermédiaire de l'offre du territoire soutient l'objectif à long terme qu'est l'amélioration de l'image du territoire.

\section{Une construction en plusieurs cercles d'implication}

L'étude comparative des processus de mise en marque des régions de l'Auvergne et de la Bretagne a permis d'identifier l'implication de parties prenantes qui se structurent en trois cercles dans lesquels la gouvernance doit trouver son équilibre. Le schéma souligne les profils d'acteurs que l'on retrouve dans les deux cas. La prise en compte des configurations locales mène à des contenus de gouvernance qui diffèrent.

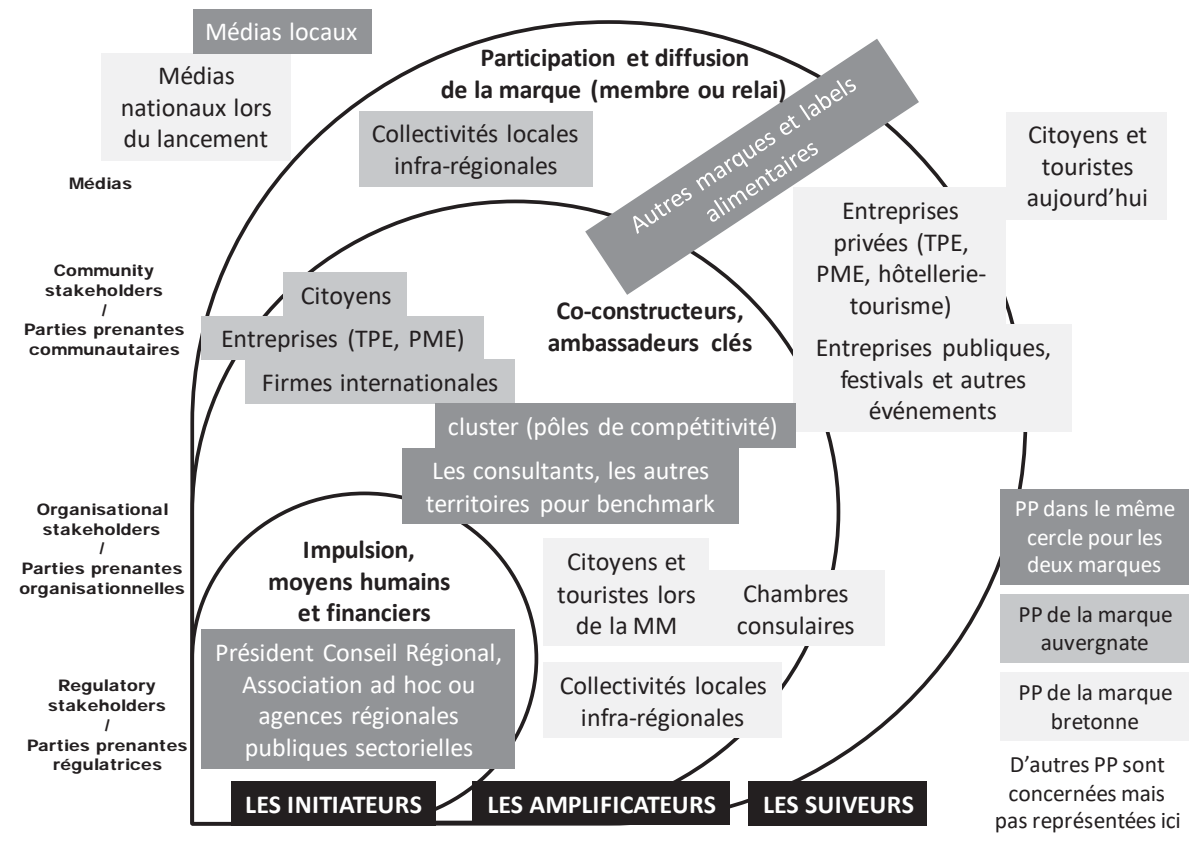

Tableau 1 - Les trois cercles des parties prenantes des deux marques Région (Bretagne et Auvergne) Source : Houllier-Guibert et Rochette (2015) 
Le premier cercle (les parties prenantes initiatrices et régulatrices) est interne à la collectivité, se dessine autour du chef de l'exécutif d'une part (instance de pilotage politique) et du collaborateur ou de la collaboratrice, désigné chef de projet (l'équipe coordinatrice qui propose les dimensions stratégiques et opérationnelles). Pour composer les instances de pilotage, la cooptation garantit la contribution des profils les plus investis. Ces instances sont nécessairement transversales :

- sur le plan politique, l'instance de pilotage regroupe minimalement autour du président, les vice-présidents les plus directement concernés par l'attractivité : économie, enseignement supérieur et recherche, tourisme, culture, urbanisme ou, dans le cas spécifique auvergnat, l'agence d'accueil des nouveaux habitants. Le portage politique incarne la marque Territoire pour laquelle il faut jouer le rôle de promoteur du territoire auprès des acteurs extérieurs;

- sur le plan technique, l'équipe coordinatrice rassemble compétences et expertises complémentaires issues des différentes directions concernées par l'attractivité. Liberté doit être accordée au chef de projet de constituer son équipe rapprochée en faisant le choix d'individualités motivées, habituées au travail en mode projet. Soulignons que le chef de projet doit avoir un profil collaboratif pour tenir le rôle central d'animation des parties prenantes, de coconstruction de la stratégie et de pilotage de la marque avec des ressources et moyens dimensionnés, dédiés à la mise en œuvre du plan d'action et au déploiement de la marque Territoire.

Ces entités de pilotage politique et technique s'attachent ensuite à emporter plus largement l'adhésion de l'institution sur le projet qui devra être présenté et expliqué aux membres des instances officielles de la collectivité : l'ensemble des élus de l'assemblée délibérante et le comité de direction réunis autour du directeur général des services. La solidité de ce premier cercle est un facteur clé de succès pour la conduite du changement. Amorcer la démarche sur la base d'objectifs partagés produit l'effet d'entraînement indispensable afin de mobiliser ensuite de façon plus large.

Une fois le premier cercle installé, le responsable politique affecté à l'initiative de la démarche de marketing territorial constitue autour de lui une instance ouverte de pilotage de l'attractivité territoriale, rassemblant acteurs publics et privés, et chargée de discuter puis de valider les orientations stratégiques. Il s'agit de rassembler les présidents des grandes institutions partenaires (consulaires, universités, technopôles, réseaux d'entreprises, offices de tourisme, rencontres d'affaires), des leaders économiques (chefs d'entreprise emblématiques du territoire, mais aussi entreprises innovantes, startup, pôles de compétitivité et autres clusters), des leaders culturels (festivals, événements, grands équipements culturels), des personnalités qualifiées, des acteurs qui disposent d'un leadership ou d'une reconnaissance dans leur secteur d'activité.

La gouvernance élargie doit favoriser l'émergence d'un dialogue simplifié et durable entre les forces vives du territoire. Elle rassemble acteurs publics et acteurs privés au service de l'élaboration et de la mise en œuvre d'une stratégie bâtie sur le socle d'une ambition commune et partagée. Cela impose de transformer les méthodes de travail, de dépasser les clivages et les contours institutionnels pour former des instances ouvertes de concertation, de réflexion, de coordination, de validation. Au regard des expériences et observations au fil de notre expertise professionnelle, nous considérons qu'il s'agit d'un travail à la fois subtil et périlleux qui nécessite l'agilité d'un funambule pour celui qui a la mission de pérenniser une gouvernance si transverse.

Les démarches de concertation rendues obligatoires dans le cadre des récentes lois sont l'occasion de rassembler les différents acteurs dans des domaines spécifiques (économie, université, numérique, innovation, tourisme). Toutes ces concertations ont comme point commun de plus ou moins se concentrer sur des focales de l'action publique quand l'attractivité du territoire - et le marketing territorial qui en découle - va concerner et mobiliser un spectre beaucoup plus large de parties prenantes. La réponse aux enjeux d'attractivité s'apparente ainsi à une métastratégie qui va inviter des acteurs de tous horizons à travailler ensemble, à discuter autour d'une même table pour partager une ambition commune, à définir ce qui rassemble et fait sens commun, à faire des choix et arrêter un positionnement, à coconstruire les fondations de la marque Territoire et l'offre qui l'incarnera. Ainsi, les démarches de marketing territorial invitent autour de la table les responsables d'un centre des congrès, d'une maison de l'emploi, d'un 
cluster, d'une université, d'un office de tourisme, d'une agence de l'innovation, d'un musée, d'une maison de la jeunesse, mais aussi des chefs d'entreprise, des étudiants, des commerçants, des hôteliers qui, ensemble, vont construire et décider du marketing à adopter.

Plusieurs mises en marque n'ont pas abouti en France faute d'avoir consacré le temps nécessaire à la mobilisation des acteurs autour d'une ambition et d'objectifs partagés en amont, ou bien faute d'orientations stratégiques communes, ou encore et le plus souvent - faute de moyens consacrés à l'animation du jeu collectif, au management du marketing territorial au moment de la mise en œuvre. Informer, expliquer et écouter les parties prenantes, les impliquer, les associer et obtenir leur engagement, puis entretenir la dynamique : ce sont les conditions à réunir pour se donner les meilleures chances de succès. La mobilisation progressive des acteurs permet de poser les bases d'un modèle pérenne de management du marketing adapté au contexte territorial.

\section{Les strates du management du marketing territorial de Brest}

L'élaboration en 2015 de la stratégie métropolitaine de développement économique a servi à mettre le territoire brestois en mouvement, à insuffler une dynamique collective autour des enjeux de développement, à mobiliser au-delà des réseaux institutionnels, et à engager un dialogue renouvelé avec les forces économiques du territoire. Cette mobilisation a profité à la mise en œuvre de la démarche de marketing territorial qui vient réinterroger les possibles coalitions de croissance de cette métropole, au-delà de la création de la marque - qui date de 2014 -, mais bien pour l'animer, la déployer, la faire exister à travers les divers leviers de l'offre du territoire. Si le cas brestois permet d'illustrer une situation qui rassemble les bases de la gestion pérenne d'une démarche d'attractivité, notamment en s'appuyant sur la gouvernance d'une stratégie globale, rappelons que chaque territoire est unique dans ses configurations et que les principes de gouvernance doivent être adaptés au regard de l'analyse du contexte.

La marque Brest Life incarne aujourd'hui la structuration de la stratégie marketing inscrite parmi les projets prioritaires de la stratégie économique brestoise. Cette démarche s'élabore autour du Comité d'orientation de la stratégie économique et installe, au gré de ses avancées, de nouvelles instances de gouvernance spécifiquement dédiées à la démarche marketing.

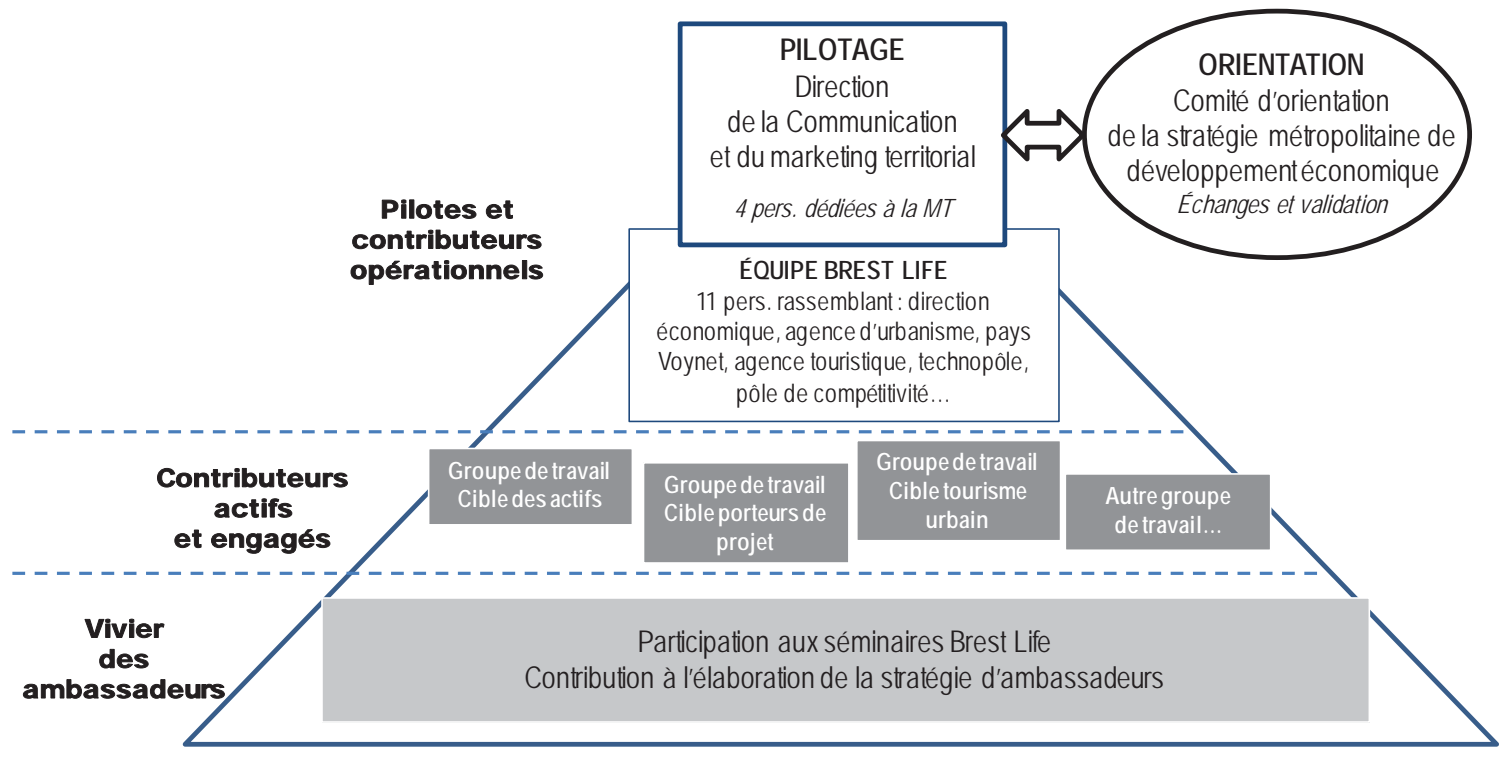

Tableau 2- La gouvernance de la marque Territoire pour manager par le développement du territoire Source : Houllier-Guibert et Miriel, 2018 


\subsection{La strate du pilotage}

Responsable du pilotage de la marque Brest L ife et plus largement de la démarche marketing, la Direction de la communication, rebaptisée Direction de la communication et du marketing territorial, s'est renforcée avec quatre personnes qui, au sein de cette direction, sont aujourd'hui directement dédiées au projet. Sur cooptation, une équipe opérationnelle restreinte, baptisée Équipe Brest Life, se réunit une fois par mois et chacun de ses membres peut être mis à contribution pour travailler dans l'intervalle des réunions. Ce groupe de travail réunit, outre les quatre personnes de la direction pilote, deux représentants de la Direction économique, le responsable des études économiques de l'agence d'urbanisme, le responsable de l'animation des développeurs économiques du Pays de Brest, le directeur de la destination touristique Brest Terres Océanes, le directeur du technopôle, un directeur de la société d'économie mixte de Brest responsable des grands équipements. L'équipe Brest Life correspond à une organisation transverse rassemblée autour de la fabrication de l'offre de territoire qui est une visée plus atteignable que l'habituelle transformation de l'image. Ses membres sont issus des entités qui contribuent historiquement au développement territorial et qui ont, par le passé, chacune à leur manière, aidé à diffuser une image dynamique du territoire et qui ont déjà créé des dispositifs d'attractivité en ciblant des publics spécifiques. Cette organisation est plus ou moins similaire à ce que l'on retrouve dans les autres régions et villes de France, avec le difficile objectif de permettre la durabilité des échanges et des constructions d'actions qui en découlent.

\subsection{La strate de la conception des dispositifs d'attractivité}

En appui de cette instance de travail opérationnelle, l'équipe responsable du projet constitue des ateliers ad hoc selon les sujets et thématiques à aborder pour élaborer la stratégie marketing et bâtir le plan d'action. Ainsi, quatre ateliers thématiques ${ }^{2}$ sur le ciblage ont été mis sur pied; ils ont accueilli chacun une douzaine de personnes, soit au total une cinquantaine d'acteurs publics et privés choisis pour leurs compétences, expertises ou domaines d'activité. Ces contributeurs actifs et engagés tirent un bénéfice de leur participation à cette dynamique, leur permettant d'élargir leur réseau, leur point de vue, leur approche du développement local. Les participants sont le plus souvent des personnalités connues pour leur dynamisme et le caractère innovant des actions qu'elles conduisent. Elles sont repérées parmi la troisième strate.

\subsection{La strate des ambassadeurs}

Environ 300 personnes constituent un groupe d'acteurs ayant manifesté leur intérêt pour la démarche de marketing territorial et qu'il convient d'animer. Il s'agit, à ce stade, d'acteurs encore relativement proches de la maittrise d'ouvrage, recensés et interpellés au cours de l'élaboration de la cartographie des acteurs qui identifie les structures puis les personnes intervenant dans les différents domaines de l'attractivité : les opérateurs satellites de la collectivité, les partenaires institutionnels, les associations incontournables, les réseaux de chefs d'entreprises, les universitaires, les responsables d'équipements et d'événements majeurs, les grandes figures culturelles et économiques. Ce vivier d'ambassadeurs est appelé à voir grossir ses rangs au fur et à mesure, à travers la stratégie de déploiement de la marque Territoire. Une sélection peut s'organiser au fil de la mobilisation des uns et des autres, avec des ambassadeurs particulièrement actifs.

Une étude récente sur les ambassadeurs de plusieurs marques Territoire en France (Houllier-Guibert et al., 2018) montre que la mobilisation des acteurs autour de la coconstruction de la stratégie est un levier essentiel pour constituer le socle du réseau d'ambassadeurs sur lequel le déploiement de la marque va pouvoir s'appuyer. Une fois la marque Territoire lancée, l'enjeu réside dans la capacité des équipes opérationnelles à animer ce réseau, à piloter le plan d'action marketing dont la mise en œuvre est partagée. Se pose alors la question des ressources et moyens alloués, sur du long terme, au déploiement marketing qui vient irriguer la majeure partie des politiques publiques, mais aussi les stratégies de développement et les actions des opérateurs partenaires de la collectivité, comme celles des entreprises, des associations culturelles, des grands événements et équipements, et potentiellement les habitants.

\section{Conclusion}

Notre propos apparait ici comme la recommandation d'une bonne pratique afin de pérenniser un marketing d'envergure basé sur une mobilisation forte des acteurs du territoire au côté des élus. À tout moment, la démarche peut être stoppée si le portage politique fait défaut, et si la mobilisation n'est pas suffisamment 
forte. Ainsi, un dispositif aussi ambitieux et qui s'inscrit dans le temps long doit réunir trois facteurs clés de succès savamment orchestrés :

- Une gouvernance basée sur les dynamiques de concertation engagées autour des schémas de développement ou sur celles de projets de territoire amorcées par les élus, ou directement installée pour la construction d'une marque.

- L'implication des membres de ces gouvernances pour qu'ils deviennent les premiers ambassadeurs. La capacité d'animation de ces partenaires directement impliqués dans la mise en œuvre du plan d'action marketing et dans le déploiement de la marque Territoire repose sur un leadership opérationnel de proximité incarné par les membres de l'équipe de coordination de la marque.

- Ce leadership doit être couplé par un second qui est plus visible. Porté par le premier élu du territoire, le leadership politique doit être associé à une vision du développement territorial à laquelle tous les acteurs sont invités à adhérer et à la traduire.

Le marketing est un état d'esprit (Levitt, 1972) et les collectivités peuvent s'en servir pour répondre aux besoins des populations résidentes afin de développer de manière durable le territoire. Une vigilance à l'égard des politiques néolibérales doit être de rigueur afin d'interroger en quoi le marketing territorial peut être aussi un outil du développement social (Noisette et Vallérugo, 2010). Cela passe par une attention particulière à propos des ciblages des politiques locales qui ne doivent pas viser strictement les populations aisées, mais continuer de prendre en compte les plus fragiles. L'étude de Pasotti dans des villes de plus grande importance que Brest, ou plus peuplées que les régions de la Bretagne et de l'Auvergne, a montré combien le marketing contribue à reléguer au second rang les enjeux de redistribution dans le cadre des politiques urbaines, à la faveur des enjeux de consommation qui seraient plus rassembleurs. Sa conclusion sur l'étude des marques de Naples, Bogota et Chicago montre des régimes urbains avec des mobilisations plutôt passives qui accordent du pouvoir aux maires et aux experts alentour. L'autre alerte de son travail porte sur la place importante que l'on accorde aux espaces centraux des villes. Ces derniers rassemblent les hauts lieux symboliques de l'histoire locale et l'ensemble des composantes qui font l'image des territoires (Houllier-Guibert, 2009) notamment, car le lien affectif passe par la pratique des espaces centraux. Mais l'offre du territoire va bien au-delà du centreville. Aussi, parmi les défis du marketing territorial, par le sentiment d'appartenance identitaire et plus largement l'image du territoire, peut être envisagée une réflexion qui consisterait à proposer des rapports avec d'autres espaces, qui concernent certaines banlieues, certains espaces périurbains, ce que le place making (Jolé et Tonnelat, 2010) ou bien les graffitis (Vaslin, 2017) permettent, par exemple. Ainsi, l'ensemble des acteurs qui font le pôle urbain serait considéré, ce qui est encore peu le cas, avec des projets très concentrés sur les centres-villes. Gageons que la taille de villes comme Brest, et plus largement la taille des villes françaises, favorise une mobilisation que les plus grandes villes du monde ne réservent qu'aux élites locales.

\section{NOTES}

1 Depuis la réforme des collectivités territoriales de 2010, une vaste transformation est mise en place progressivement en France. Dans le cadre de l'Acte III de la Décentralisation démarré en 2013, on peut identifier trois volets : la loi MAPTAM n 2014-58, crée les Métropoles qui sont 21 en 2018; la loi n 2015-29 relative à la délimitation des régions, aux élections régionales et départementales, modifie les délimitations des régions; la loi NOTRE n 2015-991 réorganise les compétences des collectivités territoriales. Cette importante réforme des périmètres de l'action publique affecte la situation des régions et des principaux pôles urbains dans le contexte national et européen ainsi que les possibilités de développement et d'aménagement, et déplace les politiques régionales vers une subsidiarité nouvelle.

2 Les quatre thèmes des ateliers portaient sur les publics cibles : actifs, porteurs de projets, étudiants/chercheurs, touristes.

\section{RÉFÉRENCES}

Houllier-Guibert, Ch.-Ed., Luisi, G., Albertini, T., Bereni, D. (2018). La mobilisation des ambassadeurs dans le management d'une marque Territoire. Etude exhaustive des régions et métropoles françaises. Revue Marketing Territorial, 1-2. Repéré à http://publis-shs.univ-rouen.fr/rmt/index.php?id=129 
Houllier-Guibert, Ch.-Ed. (2018). Proposition de cadrage définitionnel sur les marques Territoire françaises. Une question d'ambassadeurs. Revue Marketing Territorial, 1-1. Repéré à http://publis-shs.univ-rouen.fr/rmt/index.php?id=193

Houllier-Guibert, Ch.-Ed. (2012). De la communication publique vers le marketing des territoires : approche microsociologique de la fabrication de l'image de marque. Gestion et Management Public, 1-2, 35-49.

Houllier-Guibert, Ch.-Ed. (2009). Limites de la ville et image des territoires pour les communicants. Revue internationale de la communication sociale, 1, 127-144.

Jolé, M. et Tonnelat, S. (2010). Placemaking: A new approach to designing and managing urban public spaces. An interview with Kathy Madden and Fred Kent. Metropolitics, 23 (novembre).

Levitt, T. (1972). L'esprit marketing (traduction de The Marketing Mode, 1969). Paris, France : Les Éditions d'Organisation.

Noisette P., Vallerugo, F. (1996). Le marketing des villes. Un défi pour le développement stratégique. Paris, France : Les Éditions d'Organisation.

Pasotti, E. (2010). Political branding in cities. The decline of machine politics in Bogotá, Naples, and Chicago. Cambridge, Royaume-Uni: Cambridge University Press.

Pinson, G. (2011). Eleonora Pasotti. Political branding in cities. The decline of machine politics in Bogotá, Naples, and Chicago. Métropoles, 9. Repéré à http://journals.openedition.org/metropoles/4454

Vaslin, J. (2017). Esthétique propre. La mise en administration des graffitis à Paris de 1977 à 2017 (Thèse de doctorat inédite). Université de Lyon. 